\title{
Chlorella Vulgaris Biomass Supplementation Increased the Abundance of Hepatic Antioxidant Proteins in Relation to Oxidative Stress Reduction in Rabbits
}

\section{Akeem Babatunde Sikiru}

Federal University of Technology Minna

Arangasamy Arunachalam ( $\nabla$ arangasamyars@gmail.com )

ICAR-National Institute of Animal Nutrition and Physiology

Stephen Sunday Acheneje Egena

Federal University of Technology Minna

\section{Sejian Veerasamy}

National Institute of Animal Nutrition and Physiology

Ippala Janardhan Reddy

National Institute of Animal Nutrition and Physiology

Raghavendra Bhatta

National Institute of Animal Nutrition and Physiology

\section{Research}

Keywords: Oxidative stress, Chlorella vulgaris, LC-MS, Malondialdehyde, Proteins.

Posted Date: September 4th, 2020

DOI: https://doi.org/10.21203/rs.3.rs-67743/v1

License: (c) (i) This work is licensed under a Creative Commons Attribution 4.0 International License.

Read Full License 


\section{Abstract}

Background Chlorella vulgaris is a unicellular microalga that is rich in antioxidant, its supplementation has been reported to reduce oxidative stress via upregulations of antioxidant genes. However, there are scarce reports on its effect on antioxidant protein expressions in rabbits - a situation which necessitate an untargeted proteomic profile analysis due to its supplementation. This is because untargeted proteomics profiling is an approach suitable for assessing the effectiveness of genes code translation into polypeptide chains folded into functional proteins used for specific sub-cellular or extracellular physiological activities. It remains one of the comparative avenues for evaluating the efficacies of drugs and nutraceutical agents including antioxidants. In this study, the antioxidant efficacy of a microalga Chlorella vulgaris was evaluated at molecular levels using its hepatic protein expression in rabbit models.

Results After 120 days of the microalga supplementation, protein was extracted from liver of the rabbits for untargeted proteomics profiling using LC-MS/Orbitrap Fusion Tribrid ${ }^{\text {TM }}$ peptides quantifier and sequencer. There were five-hundred and eleven (511) proteins identified; and among the proteins, 191 were specific to the control group while 186 were specific to the Treatment group; and 134 were common to both groups. Independent samples t-test of the protein abundance indicated that there was a significant difference $(p=0.01)$ between the treatment and the control groups. There was also a significant reduction in the malondialdehyde concentrations $(p=0.01)$, higher total antioxidant capacities $(p=0.002)$, and increased antioxidant enzyme activities $(p=0.05)$ between the treatment and control groups.

Conclusion The study concluded that one of the molecular mechanisms associated with Chlorella vulgaris intake reduction of the hepatic oxidative stress is increased abundances of antioxidant proteins and reduction of the lipid peroxidation and these led to a suggestion that the microalga is a potent antioxidant agent suitable for protecting against oxidative stress in rabbits and other domestic food producing animals.

\section{Introduction}

Proteins are critical to normal physiological functions because they are the biomolecules enabling genes to perform their functions in an organism; hence, protecting against oxidative stress requires increased production of proteins that are specific for enhancement of the free radicals scavenging capacities of living organisms. Production of these antioxidant proteins can also be used as an indicator for determining the post-translational impacts of antioxidant agents on gene expression modulation (Calhoun and Kwon, 2011). Furthermore, quantification and identification of proteins concerning antioxidant agent supplementation could also serve as a novel approach for tracking and understanding the oxidative stress attenuation capacity of antioxidant agents (Butterfield and Perluigi, 2017). Oxidative stress also causes changes to modulate gene expression which could as well as impact on posttranscription modifications of proteins (Allen and Tresini, 2000). 
Therefore, understanding protein expression patterns associated with antioxidant agent intake is highly desirable to confirm the potency of an antioxidant agent (Hans et al., 2008). This can also facilitate understanding the hitherto unknown protective mechanisms of antioxidant agents such as Chlorella vulgaris which has been reported to be exhibiting gene expression modulation as a mechanism in rabbit models by Sikiru et al. (2019a). Hence, in this study, an attempt has been made to assess the impact of Chlorella vulgaris supplementation on the abundance of hepatic antioxidant proteins and the serum oxidative stress biomarkers concentration and in relation with their biological functions in New Zealand White rabbit models.

\section{Materials And Methods}

\subsection{Experimental protocol and animal ethics approval}

Sixteen laboratory rabbits were used for this study and the approval for use of the animals and the implementation of the experimental protocol was obtained from a joint sitting of the Institutional Animal Ethics Committee (IAEC) of National Institute of Animal Nutrition and Physiology, India and Committee for the Purpose of Control and Supervision on Experiments on Animals (CPCSEA), India. The animals were divided into two experimental groups ( $n=8$ per group); the first group served as control while the second group served as the treatment group. The rabbits in both groups were fed ad-libitum with commercial rabbit feed (Krishna Valley Agrotech, India) while rabbits in the treatment group in addition to the basal feed were supplemented with $500 \mathrm{mg}$ Chlorella vulgaris biomass per kg body weight. Water was supplied ad-libitum through automatic nipple drinkers, the light regime was 12 - 16 hours daily, and the rabbits were housed individually in their hutches while the experimental study lasts for 120 days.

\subsection{Samples preparation and protein extraction}

2.2.1 Sample collection and storage: approximately $200 \mathrm{mg}$ of liver samples of each rabbit in both groups was obtained in triplicate into cold phosphate buffer $\mathrm{pH} 7.4$ and stored ahead of downstream proteomics profiling analysis at $-80^{\circ} \mathrm{C}$ (Thermo Fisher Scientific, India).

2.2.2 Estimation of protein concentrations in the samples: Homogenates of liver tissues were prepared and concentration of protein in each homogenate was determined using Modified Biuret End Point Assay (Arkray Healthcare (P) Ltd., India). The assay principle involves the reaction of protein with cupric ions in alkaline solutions to form coloured chelate which has its absorbance measured at $578 \mathrm{~nm}$. The assay comprised of Biuret reagent made up of Copper sulfate $(7 \mathrm{mM} / \mathrm{L})$, Sodium hydroxide ( $200 \mathrm{mM} / \mathrm{L})$, Sodium-Potassium Tartrate $(20 \mathrm{mM} / \mathrm{L})$ and a surfactant. Bovine serum albumin was used as standard and the reactions were incubated at $37^{\circ} \mathrm{C}$ for 5 minutes, after which the absorbance of the samples was measured using Thermo Multiskan GO microplate reader (Thermo Fisher Scientific, Finland).

2.2.3 Determination of oxidative stress biomarkers: Oxidative stress biomarkers including malondialdehyde, protein carbonyl contents, total antioxidant capacity, and antioxidant enzyme activities; 
all these biomarkers were determined using chemical assays as described in previous studies by Sikiru et al. (2019a).

\subsection{Proteomic profiling: protein extraction, precipitation, digestion, and quantification}

2.3.1 Protein extraction: Protein extraction from the liver of the rabbits: proteins were extracted from the rabbits liver samples using Radioimmunoprecipitation assay (RIPA) buffer made up of $25 \mathrm{mM}$ Tris $\mathrm{HCl}$ ( $\mathrm{pH} 7.6), 150 \mathrm{mM}$ sodium chloride $(\mathrm{NaCl}), 1 \%$ nonyl phenoxypolyethoxylethanol -40 (NP - 40), and 0.1 $\%$ sodium dodecyl sulfate(SDS). The samples earlier stored at $-80^{\circ} \mathrm{C}$ were then thawed overnight at 4 ${ }^{\circ} \mathrm{C}$, washed in phosphate buffer ( $\mathrm{pH}$ 7.4). For each of the samples, $100 \mathrm{mg}$ were lysed under liquid nitrogen after which they were homogenized for 1 minute using the RIPA buffer and sonicated for 80 seconds in 4 strokes of 15 seconds each at 5 seconds interval (Thermocube, SScooling Systems; USA). The samples after sonication were centrifuged at $10000 \mathrm{rpm}$ at $4{ }^{\circ} \mathrm{C}$ for 10 minutes (REMI microfuge, India) and the supernatants were collected and stored at $-80^{\circ} \mathrm{C}$ ahead of the proteomic analysis.

2.3.2 Protein precipitation, digestion, and desalting: The protein samples approximately $20 \mu \mathrm{g}$ were precipitated in acetone overnight then centrifuge at $10000 \mathrm{rpm}$ (REMI Microfuge, India) and dissolved in $25 \mathrm{mM}$ ammonium bicarbonate; the dissolved proteins were then used for analysis. The analysis, commenced with the addition of $100 \mathrm{mM}$ of DTT (dithiothreitol) then incubated at $37^{\circ} \mathrm{C}$ for 30 minutes. After the incubation, there was addition of $100 \mathrm{mM}$ IAA (lodoacetamide) to obtain a concentration of 30 $\mathrm{mM}$ in the solution and kept in dark for 30 minutes after which the $\mathrm{pH}$ of the solution was adjusted to 8 by adding $25 \mathrm{mM}$ of ammonium bicarbonate. This was followed by digestion in trypsin $(0.2 \mu \mathrm{g} / \mu \mathrm{L})$ at a concentration of 1:50 trypsin: proteins; and after the trypsin addition, the samples were incubated overnight at $37^{\circ} \mathrm{C}$. Formic acid (final concentration of $0.1 \%$ in the solution) was then added to stop the reaction. The digested protein samples were desalted using $3 X$ of $100 \%$ Acetonitrile and $3 X$ of $0.1 \%$ formic acid in a mini-column, which wets the resin and conditions it to be ready to bind peptides.

2.3.3 LC-MS quantification: For peptides quantification, $1 \mu \mathrm{L}$ of the digested samples were used for LCMS quantification of the peptides in the protein samples using LC Model EASY nLC 1200 (Thermo Fisher Scientific, USA) with nanoflow rate $300 \mathrm{~nL} / \mathrm{min}$ and buffers including two buffers ( $0.1 \%$ Formic acid in HPLC water and $80 \%$ Acetonitrile $+0.1 \%$ formic acid in HPLC water). The LC gradient and MS measurements of the quantification are presented in Tables 1 and 2 respectively.

Table 1: The LC gradient method used for the quantification of the peptides in the protein samples 


\begin{tabular}{|lll|}
\hline Time (mins) & Flow (nL/min) & Percentage of the buffer \\
\hline 0.00 & 300 & 10 \\
\hline 02.00 & 300 & 15 \\
\hline 50.00 & 300 & 45 \\
\hline 52.00 & 300 & 98 \\
\hline 60.00 & 300 & 98 \\
\hline
\end{tabular}

Table 2: Details of mass spectroscopy used in the quantification of the protein peptides in the samples

\begin{tabular}{|ll|}
\hline Parameters & \\
\hline Detector type & Orbitap \\
\hline Resolution & 120000 \\
\hline RF lens & $60 \%$ \\
\hline Ion polarity & Positive \\
\hline MS Scan range (m/Z) & $375-1700$ \\
\hline Maximum injection time (min) & 50 \\
\hline
\end{tabular}

\section{Data Analysis And Evaluation Of Relative Protein Abundance}

The uniprot_rabbit_2019 database was used for protein sequence identification; the fragment mass tolerance used was $0.6 \mathrm{Da}$ and the false discovery rate (FDR) used was $1 \%$ at the significance threshold of $p<0.004$; for the taxonomy of the proteins sequences, all possible entries of the amino acids were accepted. Chlorella vulgaris supplementation effects on oxidative stress biomarkers and the abundance of commonly expressed proteins were determined using independent samples t-test at $p<0.05$ level of probability. There was the determination of interactions among the significantly upregulated antioxidant and stress-related proteins using the STRING database while functional enrichment analysis of their associated pathways was carried out using g:profiler which is a web server for functional enrichment analysis and conversions of gene lists (Raudvere et al., 2019).

\section{Results}

In total, there were 511 proteins identified, and out of these proteins, 191 proteins were specific to the control group while 186 proteins were specific to the treatment group and a total of 134 proteins were 
common to both the control and treatment groups (Fig. 1). The detailed descriptions of all the proteins identified in each of the groups are presented as supplementary data (S1).

The records of oxidative stress biomarkers and protein abundance were subjected to Levene's test of variance equality and this revealed there were significant differences in both oxidative stress biomarkers and protein abundance in serum and liver of the rabbits respectively. The Chlorella vulgaris supplementation significantly affected the abundance of commonly expressed proteins in the liver samples, the Treatment group was $2.58 \pm 0.60 \mathrm{emPAl}$ compared with Control group which was $1.09 \pm 0.44$ emPAl $(p=0.01)$; while the minimum and maximum abundance of the commonly expressed proteins were 0.16 and 2.30 emPAl respectively. There was a higher abundance of antioxidant proteins in the treatment group compared with the control group (Fig. 2). There were interactions between significantly abundant antioxidant proteins and a multi-query Manhattan plot of the interactions between the functionally enriched pathways associated with the significantly abundant proteins using g:profiler showed that these proteins were associated with 41 pathways associated with the processes of oxidative stress attenuation and antioxidant activities of free radicals scavenging (Fig. 3).

The mean liver malondialdehyde concentrations of the rabbits (MDA) was $23.90 \pm 1.65$ and $16.77 \pm$ $1.34 \mu \mathrm{mol} / \mathrm{mL}$ for the control and treatment groups, respectively $(p=0.01)$, liver protein carbonyl contents (PCO) was $7.74 \pm 0.55$ and $6.22 \pm 0.03 \mu \mathrm{mol} / \mathrm{mL}$ for the control and treatment groups, respectively $(\mathrm{p}=$ 0.06 ), while liver total antioxidant capacity (TAC) was $3.79 \pm 0.44$ and $8.03 \pm 0.50 \mu \mathrm{mol} / \mathrm{mL}$ for the control and treatment groups, respectively $(p=0.002)$. There was increased activities of antioxidant enzymes in the treatment group and these corresponded with a reduced concentration of oxidative stress biomarkers as well as an increased abundance of proteins in the treatment group compared with control (Figs. 4 and $5)$.

\section{Discussion}

Oxidative stress is a biochemical process causing the generation of reactive oxygen species and any other species, molecules, and or ions formed through incomplete electron reduction reaction of oxygen. It is involved in pathways affecting functional cellular biomolecules including lipids, and these are factors leading to progression of disease conditions, including male infertility, pulmonary carcinogenesis, hypoxia, and vascular malfunctions in humans (Kalyanaraman et al., 2018; Mennerich et al., 2019; Baker et al., 2019; Tejero et al., 2019; Valavanidis, 2019;). Although supplementation of antioxidants has been widely reported as a way-out for oxidative stress protection, the mechanisms underlying their involvement in oxidative stress protection in-vivo is poorly understood (Hans et al., 2008). However, recent investigations on antioxidant agent supplementation identified gene expression and regulation modification for cellular protection against oxidative stress in rabbits and other models as mechanisms associated with oxidative stress (Ahmed et al., 2016; Dornas et al., 2017; Sikiru et al. 2019b).

This current study confirmed some of these assertions that Chlorella vulgaris biomass as an antioxidant agent has post-transcription modulation impacts against oxidative stress because its supplementation 
led to a higher abundance of antioxidant proteins in rabbit models. This outcome showed that the primary antioxidant enzymes such as superoxide dismutase could be active across both the cytoplasm and mitochondria phases of oxidative stress protections since their proteins were more abundant in the treatment group versus control and the proteins are associated with cytosolic and mitochondria protection against oxidative stress (Song et al., 2017).

Furthermore, functional enrichment of the proteins indicated their connections with several other proteins involved in the scavenging of free radicals. This is an effect which indicated that Chlorella vulgaris is a potential drug and nutraceutical candidate for oxidative stress attenuation owing to the importance of the pathways it modulated in response to cellular oxidative stress via the hydrogen peroxide metabolism and detoxification of xenobiotics compounds. These cellular activities can be linked to prominent mechanisms involving catalytic removal of free radicals by superoxide dismutase, catalase, and peroxidase; and scavenging of reactive species by low molecular agents such as carotenoids present in the microalga (Barber et al., 2006). Superoxide dismutase proteins were more in abundance in the supplemented rabbits, and this could be regarded as the starting point of endogenous antioxidant protection whereby one superoxide radical is oxidized and the second one is reduced. This leads to the production of hydrogen peroxide and oxygen; the hydrogen peroxide produced is then quickly acted upon by catalase, glutathione peroxidases in the cytoplasm while in the mitochondria glutathione reductase inhibit the activities of free radicals (Barber et al., 2006).

The supplementation of Chlorella vulgaris as an antioxidant in this study as revealed in the interactions and functional enrichment indicated that its supplementation to the rabbit led to increased activities of the antioxidant enzymes and increased production of their respective proteins to prevent oxidative stress progression in a mechanism similar to impacts of ascorbate as an antioxidant agent (Halliwell and Gutteridge, 1999). Catalase (CAT), peroxiredoxin (PRDX6), macrophage migration inhibitory factor (MIF), and Glutathione S-Transferase Pi 1 (GSTP1) were all the antioxidant protein higher in their abundance in the supplemented group compared with the control animals in this study.

In relating these abundances with reduction of the oxidative stress biomarker (malondialdehyde) which was observed in the supplemented rabbit, it could be inferred that the increased expression of these proteins as a mechanism of oxidative stress protection in the rabbits is promoted by the microalga intake. Catalase is one of the proteins produced in higher quantity in the supplemented rabbits, this is a protein whose expression could lead to the protection of cells from the toxic effects of hydrogen peroxide as well as promoting the growth of immune cells, including T-cells and B-cells, and hence, could be linked with double effects of oxidative stress protection and immune enhancement. This observation is in agreement with Perl (2013), who stated that antioxidant therapy focusing on reducing oxidative stress and enhancing activities of T-cells and B-cells could contribute to immune protection against oxidative stress-mediated diseases such as organ damage and comorbidities in patients with systemic lupus erythematosus (SLE). 
Similarly, increased expression of peroxiredoxin (PRDX6) in the supplemented rabbit affirm competency of the microalga supplemented to protect against oxidative stress. This is because as a protein encoded gene and a member of the thiol-specific antioxidant protein family, it has a bifunctional enzyme with two distinct active sites involved in redox regulation of the cell (Manevich and Fisher, 2005). The increased expression of peroxiredoxin in the supplement rabbits can reduce pro-oxidant activities of hydrogen peroxide and short-chain organic, fatty acid, and phospholipid hydroperoxides and it may also play a role in the regulation of phospholipid turnover as well as in protection against oxidative injury (Fujii and Ikeda, 2002). The microalga supplementation also increased macrophage migration inhibitory factor which indicates that it is a potent antioxidant agent since the gene MIF encodes a lymphokine involved in cellmediated immunity, immunoregulation, and inflammation which plays roles in the regulation of macrophage function in host defense through the suppression of anti-inflammatory effects of glucocorticoids - a mechanism substantial for protection against environmental triggered stress at the cellular level (Park, 2018).

The results from this study also indicated that the microalga supplementation led to a reduction in the serum malondialdehyde and protein carbonyl with a corresponding increase in the serum total antioxidant capacities of the rabbits in the Treatment group. These observations recorded indicated that the supplemented microalga prevented lipid peroxidation in the rabbit because of the reduction in the serum malondialdehyde concentration. The biochemical processes associated with production, metabolism, and signaling functions mediated by malondialdehyde revealed that it increasing production is linked with several pathological developments in a given biological system (Ayala et al., 2014). The mechanism of malondialdehyde compromising of physiological activities cut-across cells, tissues, organs, and the entire organism because it negatively affects lipid signaling pathways such as the activities of steroid hormones including oestrogen, testosterone, and cortisol, which are responsible for the modulation of a host organism functions such as reproduction, metabolism, stress response, inflammation, blood pressure, and salt and water balance (Pluchino et al., 2013).

Therefore, correlating the malondialdehyde concentration reduction and the increasing abundance of antioxidant proteins in the supplemented rabbits as observed in this study could lead to a confident juxtaposing that supplementation of the microalga Chlorella vulgaris prevented attacks on lipid signalings and also led to the upregulation of its associated genes as well as enhanced activities of the antioxidant enzymes which all led to the increased production of the antioxidant proteins. These submissions agreed with Ayala et al. (2014), which opined that under normal physiological conditions there will be reduced malondialdehyde concentrations necessary for cellular stimulation, maintenance and survival occurring through activities of antioxidant enzymes and antioxidant genes for promoting the constitutive antioxidants defense systems and protection of lipid signaling pathways as well as activation of genes expression required for upregulating antioxidants proteins needed for the formation of adequate protection and adaptation to the stress response. The underlying physiological importance of this mechanism of reducing malondialdehyde for increasing antioxidant proteins abundance is that reduced malondialdehyde concentration promotes healthy living because if otherwise, the cells can induce apoptosis or necrosis programmed cell death; and both processes will eventually lead to 
molecular cell damage and facilitation of various pathological conditions that could negatively affect normal well-being (Gudipaty et al., 2018). In conclusion, this present study in its novelty contributed that Chlorella vulgaris is a potent antioxidant agent which could be effectively used to reduce oxidative stress having observed increased abundance of antioxidant proteins and reduced serum oxidative stress products in rabbits supplemented with the microalga which suggested it is a potent antioxidant agent that could be exploited in the development of drugs for oxidative stress-related ailments.

\section{List Of Abbreviations}

CV: Chlorellavulgaris; ELU: Experimental Livestock Unit; ICAR: Indian Council for Agricultural Research; LLP: Limited Liability Private Company; PVT: Private; NIANP: National Institute of Animal Nutrition and Physiology, Bengaluru, India; IAEC: Institutional Animal Ethics Committee; MDA: Malondialdehyde; UK: United Kingdom; UV/Vis: Ultraviolet-Visible Spectrophotometry; USA: United States of America; U/mL: Units per milliliter; DEPTA: Diethylenetriaminepentaacetic acid; $\mathrm{HCl}$ : Hydrochloric acid; $\beta N A D P H: \beta-$ Nicotinamide Adenine Dinucleotide 2'-Phosphate; ANOVA: Analysis of Variance; SPSS: Statistical Package for the Social Sciences; SEM: Standard Error of Means; IBM: International Business Machines Corporation; TWAS: The World Academy of Sciences; DBT: Department of Biotechnology; CCSTDS: Centre for Co-operation in Science \& Technology among Developing Societies; SOD: Superoxide dismutase; CAT: catalase; PCO: protein carbonyl contents; TAC: total antioxidant Capacity; PRDX6: peroxiredoxin; MIF: macrophage migration inhibitory factor; GSTP1: Glutathione-S-Transferase-Pi-1; IAEC: Institutional Animal Ethics Committee; CPCSEA: Committee for Control and Supervision on Experiments on Animals; RIPA: Radioimmunoprecipitation assay; DTT: dithiothreitol; IAA: lodoacetamide; LC-MS: Liquid Chromatography-Mass Spectrometry; FDR: false discovery rate; Gol: Government of India; C-CAMP: Centre for Cellular and Molecular Platforms; emPAl: Exponentially Modified Protein Abundance Index.

\section{Declarations}

\section{Ethics and animal experimental protocol approval}

Approval of animal experimentation protocol in this study was secured from the Institutional Animal Ethics Committee (IAEC) of ICAR-National Institute of Animal Nutrition and Physiology, Bangalore, India; and Committee for the Purpose of Control and Supervision of Experiments on Animals (CPCSEA); India.

\section{Consent for publication}

Not Applicable

\section{Availability of data and materials}

Not Applicable

\section{Competing interest}


The authors declared no conflict of interest.

\section{Funding}

Sikiru A.B. received research scholarship and fellowship from the Department of Biotechnology (DBT), India; and The World Academy of Sciences (TWAS), Italy.

\section{Authors' contribution}

Sikiru A.B: carried out the animal experimentation, laboratory analysis, data analysis and draft the manuscript.

Arangasamy A. and Egena S.S.A.: supervised the experiments, validate the results and corrected the manuscript.

Sejian V., Ippala J.R. and Raghavendra B.: contributed materials for the research and corrected the manuscript draft.

\section{Acknowledgment}

The authors sincerely appreciate the support of ICAR and Director, ICAR-National Institute of Animal Nutrition and Physiology, Bengaluru; India for providing facilities used for conducting the Ph.D. research work of SAB under DBT-TWAS fellowship sponsored by the Department of Biotechnology, Government of India (Gol) and The World Academy of Sciences (TWAS), Italy. The authors also appreciate the Centre for Cellular and Molecular Platforms (C-CAMP), Bengaluru, India, for providing facilities used for protein profiling and analysis of the LC-MS results.

\section{References}

1. Ahmed AA, Fedail JS, Musa HH, Musa TH, Sifaldin AZ. Gum Arabic supplementation improved antioxidant status and alters expression of oxidative stress gene in ovary of mice fed high fat diet. Middle East Fertility Society Journal. 2016 Jun 1;21(2):101-8.

2. Allen RG, Tresini M. Oxidative stress and gene regulation. Free Radical Biology and Medicine. 2000 Feb 1;28(3):463-99.

3. Ayala A, Muñoz MF, Argüelles S. Lipid peroxidation: production, metabolism, and signaling mechanisms of malondialdehyde and 4-hydroxy-2-nonenal. Oxidative medicine and cellular longevity. 2014 Oct;2014.

4. Baker MA, Netherton J, Aitken RJ. From past to present: an historical overview of the concept of spermatozoa, reactive oxygen species, and male-factor infertility. InOxidants, Antioxidants and Impact of the Oxidative Status in Male Reproduction 2019 Jan 1 (pp. 17-26). Academic Press.

5. Barber SC, Mead RJ, Shaw PJ. Oxidative stress in ALS: a mechanism of neurodegeneration and a therapeutic target. Biochimica et Biophysica Acta (BBA)-Molecular Basis of Disease. 2006 Nov 
1;1762(11-12):1051-67.

6. Butterfield DA, Boyd-Kimball D, Castegna A. Proteomics in Alzheimer's disease: insights into potential mechanisms of neurodegeneration. Journal of neurochemistry. 2003 Sep;86(6):1313-27.

7. Butterfield, D. Allan, and Marzia Perluigi. Redox proteomics: a key tool for new insights into protein modification with relevance to disease. (2017): 277-279.

8. Calhoun LN, Kwon YM. Structure, function and regulation of the DNA-binding protein Dps and its role in acid and oxidative stress resistance in Escherichia coli: a review. Journal of applied microbiology. 2011 Feb;110(2):375-86.

9. De Henau S, Tilleman L, Vangheel M, Luyckx E, Trashin S, Pauwels M, Germani F, Vlaeminck C, Vanfleteren JR, Bert W, Pesce A. A redox signalling globin is essential for reproduction in Caenorhabditis elegans. Nature communications. 2015 Dec 1;6(1):1-4.

10. Dornas WC, Cardoso LM, Silva M, Machado NL, Chianca-Jr DA, Alzamora AC, Lima WG, Lagente V, Silva ME. Oxidative stress causes hypertension and activation of nuclear factor-kB after highfructose and salt treatments. Scientific reports. 2017 Apr 11;7:46051.

11. Fujii J, Ikeda Y. Advances in our understanding of peroxiredoxin, a multifunctional, mammalian redox protein. Redox Report. 2002 Jun 1;7(3):123-30.

12. Gudipaty SA, Conner CM, Rosenblatt J, Montell DJ. Unconventional ways to live and die: cell death and survival in development, homeostasis, and disease. Annual review of cell and developmental biology. 2018 Oct 6;34:311-32.

13. Halliwell B, Gutteridge JM. Free radicals in biology and medicine. Oxford University Press, USA; 2015.

14. Kalyanaraman B, Cheng G, Hardy M, Ouari O, Bennett B, Zielonka J. Teaching the basics of reactive oxygen species and their relevance to cancer biology: Mitochondrial reactive oxygen species detection, redox signaling, and targeted therapies. Redox biology. 2018 May 1;15:347-62.

15. Manevich Y, Fisher AB. Peroxiredoxin 6, a 1-Cys peroxiredoxin, functions in antioxidant defense and lung phospholipid metabolism. Free Radical Biology and Medicine. 2005 Jun 1;38(11):1422-32.

16. Mennerich D, Kellokumpu S, Kietzmann T. Hypoxia and reactive oxygen species as modulators of endoplasmic reticulum and Golgi homeostasis. Antioxidants \& redox signaling. 2019 Jan 1;30(1):113-37.

17. Park M. Dissecting the impact of macrophage migration inhibitory factor (MIF) on host immune response (Doctoral dissertation, Virginia Tech).

18. Perl A. Oxidative stress in the pathology and treatment of systemic lupus erythematosus. Nature Reviews Rheumatology. 2013 Nov;9(11):674.

19. Pluchino N, Russo M, Santoro AN, Litta P, Cela V, Genazzani AR. Steroid hormones and BDNF. Neuroscience. 2013 Jun 3;239:271-9.

20. Raudvere U, Kolberg L, Kuzmin I, Arak T, Adler P, Peterson H, Vilo J. g: Profiler: a web server for functional enrichment analysis and conversions of gene lists (2019 update). Nucleic acids research. 2019 Jul 2;47(W1):W191-8. 
21. Sikiru AB, Arangasamy A, Alemede IC, Egena SS, Bhatta R. Dietary supplementation effects of Chlorella vulgaris on performances, oxidative stress status and antioxidant enzymes activities of prepubertal New Zealand White rabbits. Bulletin of the National Research Centre. 2019 Dec 1;43(1):162.

22. Sikiru AB, Arangasamy A, Alemede IC, Guvvala PR, Egena SS, Ippala JR, Bhatta R. Chlorella vulgaris supplementation effects on performances, oxidative stress and antioxidant genes expression in liver and ovaries of New Zealand White rabbits. Heliyon. 2019 Sep 1;5(9):e02470.

23. Song X, Narzt MS, Nagelreiter IM, Hohensinner P, Terlecki-Zaniewicz L, Tschachler E, Grillari J, Gruber F. Autophagy deficient keratinocytes display increased DNA damage, senescence and aberrant lipid composition after oxidative stress in vitro and in vivo. Redox biology. 2017 Apr 1;11:219-30.

24. Stadtman ER, Levine RL. Chemical modification of proteins by reactive oxygen species. Redox proteomics: from protein modifications to cellular dysfunction and diseases. 2006 Aug 11;9(3).

25. Tejero J, Shiva S, Gladwin MT. Sources of vascular nitric oxide and reactive oxygen species and their regulation. Physiological reviews. 2019 Jan 1;99(1):311-79.

26. Valavanidis A. Oxidative Stress and Pulmonary Carcinogenesis Through Mechanisms of Reactive Oxygen Species. How Respirable Particulate Matter, Fibrous Dusts, and Ozone Cause Pulmonary Inflammation and Initiate Lung Carcinogenesis. InOxidative Stress in Lung Diseases 2019 (pp. 247265). Springer, Singapore.

\section{Figures}




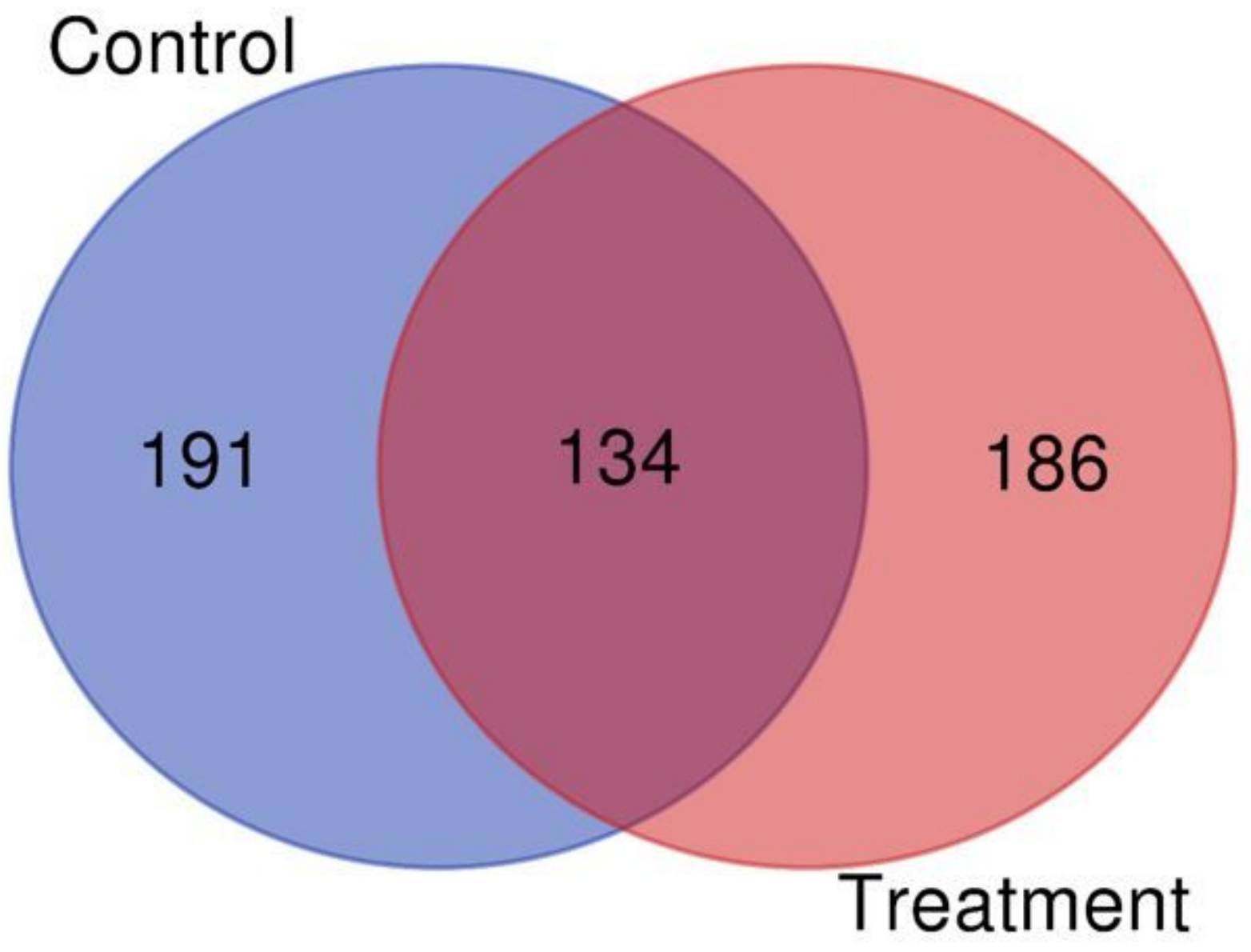

Figure 1

Abundance of the expressed proteins in the liver of the New Zealand White rabbits. Control - No supplementation. Treatment - Rabbits supplemented with $500 \mathrm{mg}$ Chlorella vulgaris biomass per $\mathrm{kg}$ body weight daily. 


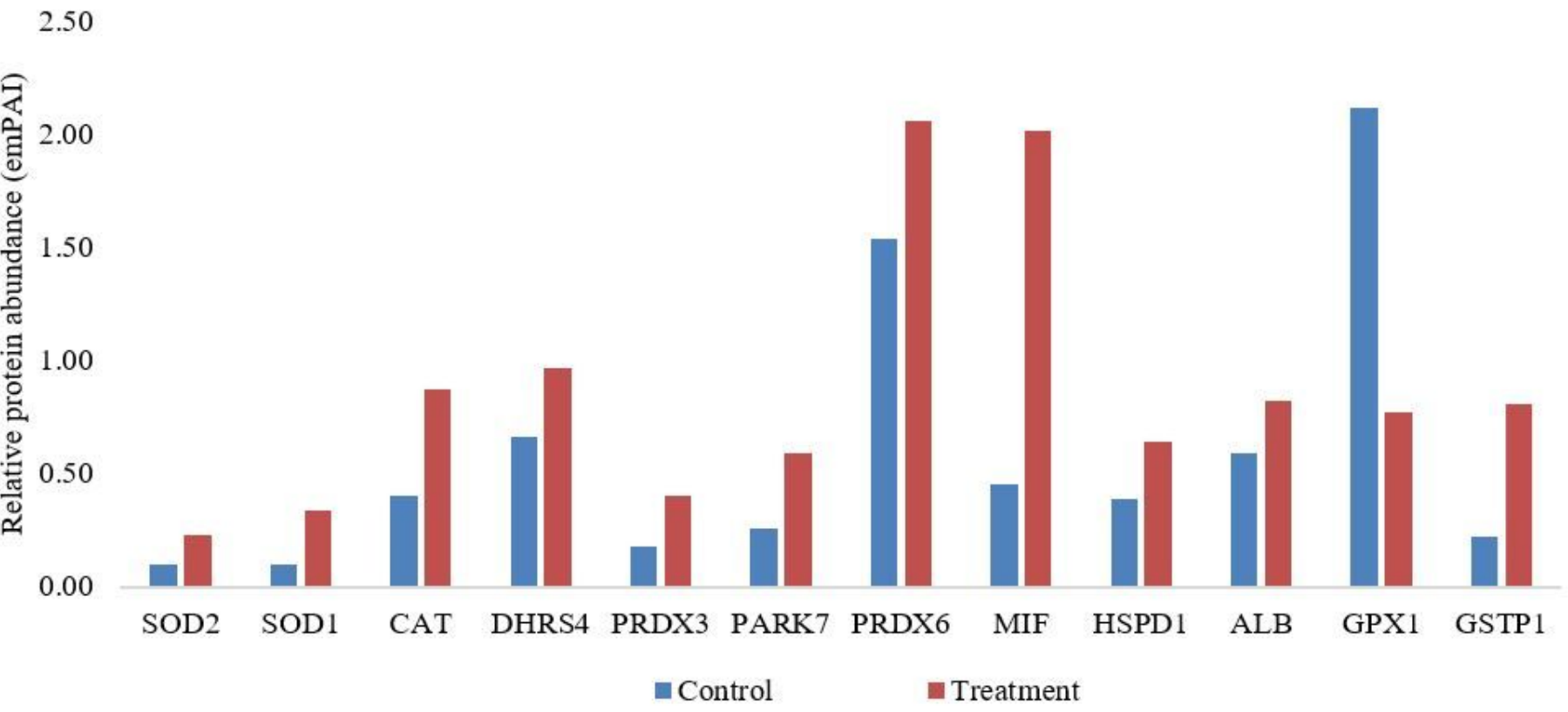

Figure 2

Comparative abundance of antioxidant and stress-related proteins in the liver of New Zealand White rabbits supplemented with microalga Chlorella vulgaris biomass. Control - No supplementation.

Treatment - Rabbits supplemented with $500 \mathrm{mg}$ Chlorella vulgaris biomass per kg body weight of the rabbits.

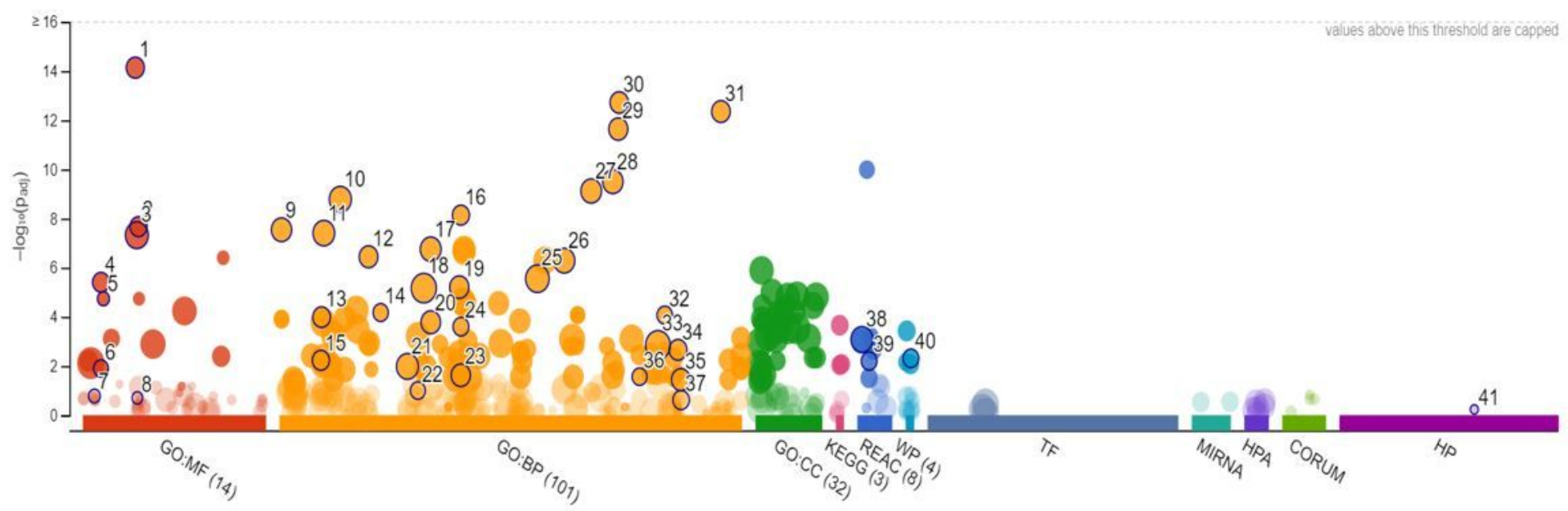

Figure 3

Multiquery Manhattan plot of the functionally enriched pathways associated with the significantly abundant proteins in the rabbits supplemented with Chlorella vulgaris biomass at $500 \mathrm{mg} / \mathrm{kg}$ body weight per day. 1. GO:0016209. 2. G0:0016684. 3. G0:0016491. 4. GO:0004601. 5. G0:0004784. 6. GO:0004602. 7. GO:0004096. 8. G0:0016532. 9. GO:0000302. 10. GO:0009636. 11. GO:0006979. 12. G0:0016999. 13. G0:0006801. 14. G0:0019430. 15. GO:0006749. 16. G0:0042743. 17. G0:0034599. 18. 
GO:0033554. 19. G0:0042542. 20. G0:0034614. 21. GO:0031667. 22. G0:0032928. 23. G0:0042737. 24. GO:0042744. 25. G0:0055114. 26. G0:0062197. 27. GO:0072593. 28. G0:0097237. 29. G0:0098754. 30. GO:0098869. 31. GO:1990748. 32. G0:1902175. 33. GO:1901700. 34. GO:1903201. 35. G0:1903409. 36. GO:1900409. 37. GO:1903427. 38. REAC:R-HSA-2262752. 39. REAC:R-HSA-9615017. 40. WP:WP408. 41. HP:0012517.

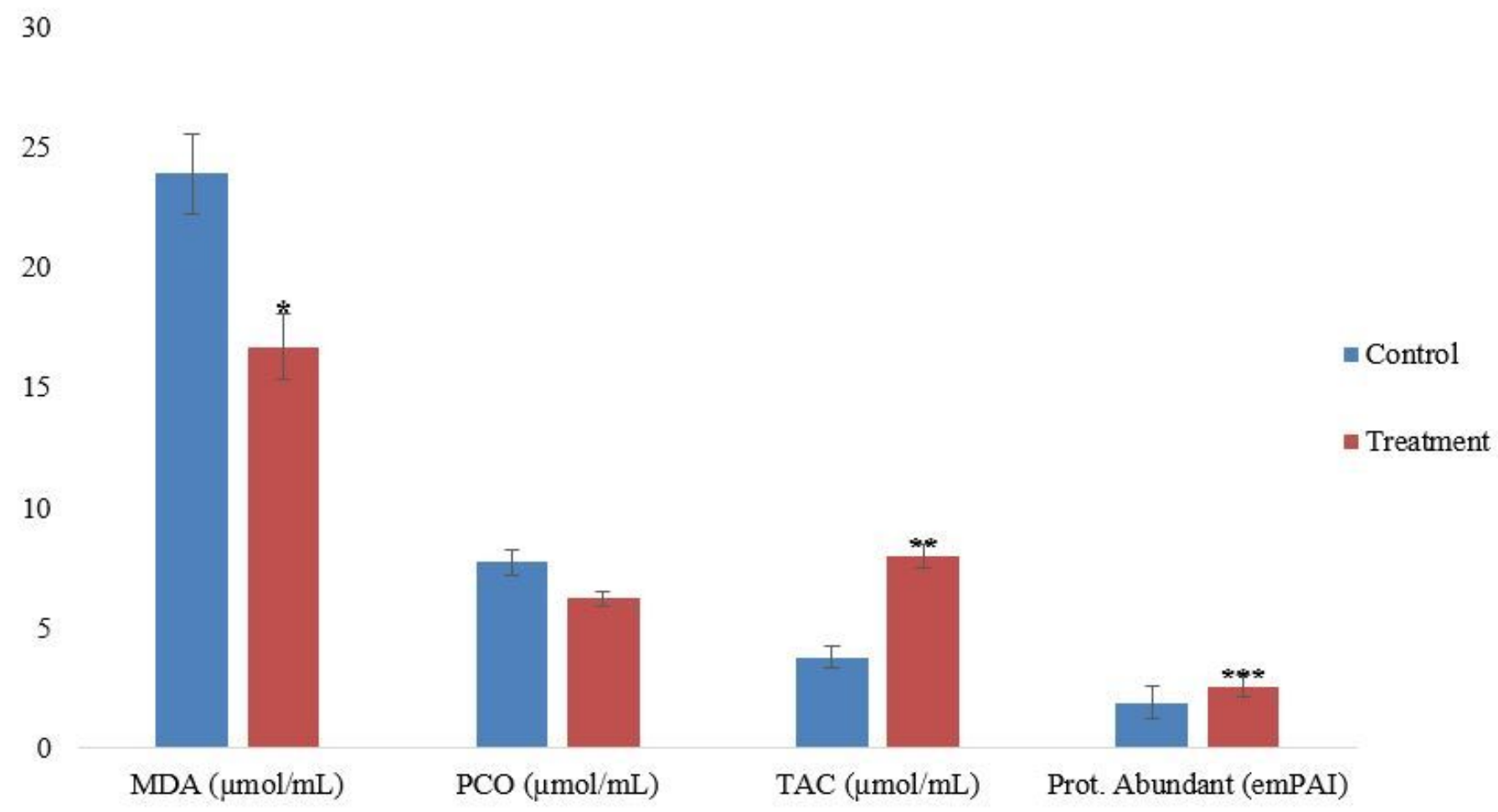

\section{Figure 4}

Comparative effects of Chlorella vulgaris supplementation on liver oxidative stress biomarkers and abundance of commonly expressed proteins in the rabbits. Control - No supplementation. Treatment Rabbits supplemented with $500 \mathrm{mg}$ Chlorella vulgaris biomass per $\mathrm{kg}$ body weight of the rabbits. Bars with different labels are significantly different $(p<0.05)$ for each of the parameters determined. 
6

4

2

0

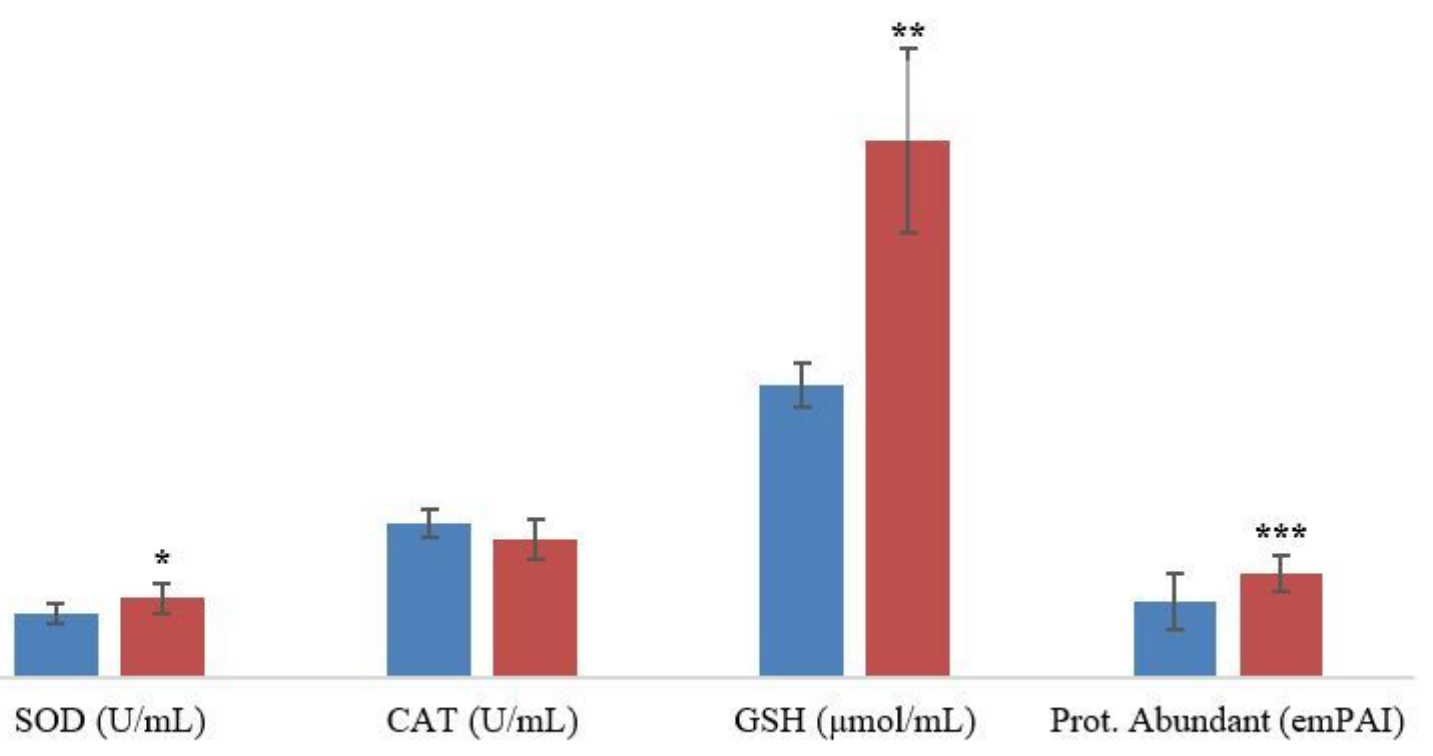

- Control

- Treatment

\section{Figure 5}

Effects of Chlorella vulgaris supplementation on liver antioxidant enzyme activities and abundance of commonly expressed proteins in the rabbits. Control - No supplementation. Treatment - Rabbits supplemented with $500 \mathrm{mg}$ Chlorella vulgaris biomass per kg body weight of the rabbits. Bars with different labels are significantly different $(p<0.05)$ for each of the parameters determined.

\section{Supplementary Files}

This is a list of supplementary files associated with this preprint. Click to download.

- Highlights.docx

- Highlights.docx

- GraphicalAbstract.pptx

- GraphicalAbstract.pptx

- Proteinabundance.xlsx

- Proteinabundance.xlsx 